\title{
Pflegekombination bei Gesichtsrötungen
}

Gerötete Haut und rote Äderchen sind oftmals auch Begleiterscheinungen von Rosazea und Couperose. Mehr als vier Millionen Menschen leiden unter den auffälligen roten Flecken oder auch erweiterten Äderchen. Dermasence bietet mit „den starken Zwei“ eine gezielte Pflegekombination, die ideal auf die zu Rötungen neigende Gesichtshaut abgestimmt ist.

Als Intensivpflege erzielt das Dermasence Refining Gel mit dem 6-fachPflegekomplex eine gefäßverengende Wirkung. Es fördert die Stabilität der Gefäße und lässt erweiterte Äderchen blasser werden. Besonders wichtig ist es, die Entzündungskaskade $\mathrm{zu}$ senken. Viele Rosazea-Patienten leiden an einer Fehlregulation der Cathelicidin-Expression, was gleichzeitig mit einer überschie-

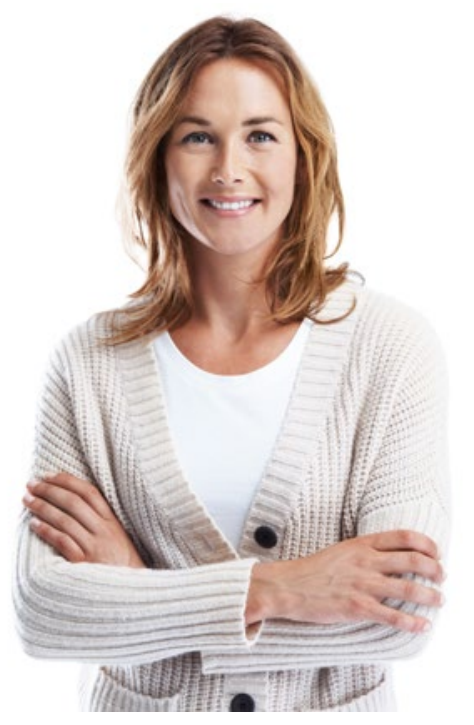

Eine konsequente Pflege kann Rötungen und Irritationen sichtbar reduzieren. ßenden Entzündungsreaktion einhergeht. $2 \times$ täglich angewandt, kann das Gel diese Entzündungskaskade bei Rosazea nachhaltig drosseln - mit einem 24 hEntzündungsschutz.

Dermasence Cream Soft ist die ideale Ergänzung zur Tages- und Nachtpflege. Die leichte Gelcreme setzt Feuchtigkeit auf der Haut frei und kühlt zusätzlich. Das Hautbild wird durch Panthenol und Bisabolol beruhigt. Nicht komedogene Naturöle nehmen das unangenehme Spannungsgefühl.

Bei regelmäßiger Anwendung „der starken Zwei“" wird das Hautbild verfeinert, Rötungen werden dauerhaft gemindert. Die überzeugende Pflegekombination ist ab sofort als praktisches Set erhältlich. Patienten-Informationshandzettel (DIN A5) und Themenfolder „Rosacea“ können kostenlos über den Dermasence-Außendienst oder telefonisch unter 0251/91599-100 angefordert werden.

Nach Informationen von P\&M Cosmetics

Ergebnisse aus der Praxis belegen Wirksamkeit

\section{Narbenbehandlung mit Intensivpatch für die Nacht}

\begin{abstract}
Montanari et al. stellten auf dem diesjährigen IMCAS(International Master Course on Aging Skin)-Kongress in Paris aktuelle Ergebnisse aus der ärztlichen Praxis zur Anwendung eines Intensivpatches vor. In der strukturierten Kasuistikauswertung mit 34 Patienten (61,8\% Frauen, Durchschnittsalter 43,8 Jahre) wurde die Effizienz und Verträglichkeit
\end{abstract}

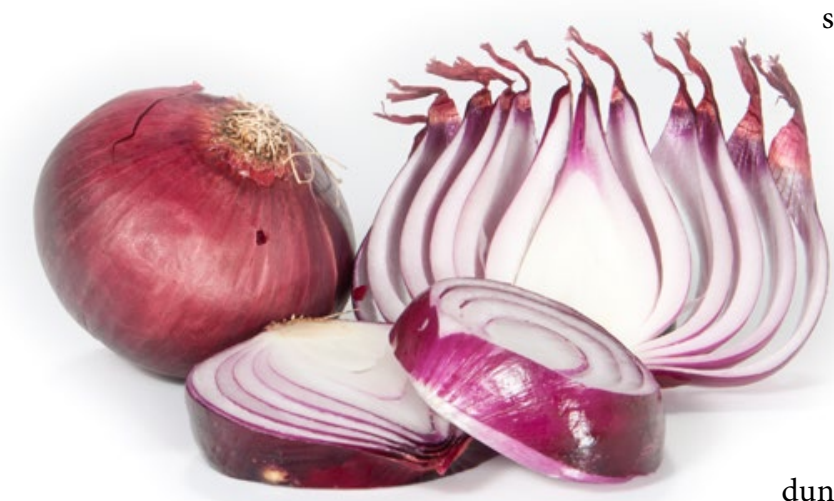

des Narbentherapeutikums getestet. Das Intensivpatch wird über Nacht angewandt und zeichnet sich durch eine duale Wirkweise aus. Mittels Okklusion und gleichzeitiger Freisetzung der Inhaltsstoffe Cepalin und Allantoin wird die Narbenbildung reguliert. Über einen Zeitraum von 3 Monaten erfolgte die Behandlung von Patienten mit postoperativen Narben in 12 dermatologischen und chirurgischen Praxen in Deutschland. Das Alter der Narben variierte von 5 Tagen bis zu 7 Jahren, wobei 42,4\% der Narben aus Muttermalentfernungen resultierten und $15,5 \%$ aus kosmetischen Eingriffen. Bei $85,3 \%$ der Patienten erfolgte die Behandlung mit dem Intensivpatch als Ersttherapie. Die Anwendung des Intensivpatches erfolgte Auch die Leitlinien führen Extractum-cepaehaltige Externa zur Narbentherapie auf. für 6-12 Stunden über Nacht auf das Narbengewebe aufgelegt.
Nach dreimonatiger Behandlung berichteten 93,5\% der Patienten von mindestens zwei positiven Entwicklungen des Narbengewebes. Insbesondere wurde eine verbesserte Ebenmäßigkeit der Narbe und keine oder geringe Hypertrophie der Narbe erzielt. Zudem entwickelten sich die Elastizität und Weichheit der Narbenstruktur positiv. In 35,5\% der Fälle reduzierte sich außerdem die Rötung der therapierten Narbe. Lediglich 6 der 34 behandelten Patienten berichteten unerwünschte Wirkungen, die nicht schwerwiegend und auf die Narbe lokalisiert waren.

In den aktuellen internationalen sowie deutschen Leitlinien zur Narbentherapie werden sowohl die positiven Effekte von Okklusion als auch von Extractum-cepae-haltigen Externa zur Narbenprophylaxe und Narbentherapie herausgestellt.

Das Contractubex ${ }^{\oplus}$ Intensivpatch bedient sich einer dualen Wirkweise aus Okklusion und Freisetzung der Inhaltsstoffe Cepalin (Extractum Cepae) und Allantoin über mehrere Stunden. Die empfindliche Narbenregion wird nach Applikation des Patches durch das dünne Schaummaterial bedeckt und sorgt so für eine feuchtigkeitserhaltende Atmosphäre im behandelten Hautbereich.

Nach Informationen von Merz Pharmaceuticals 